\title{
Nomadismo, heterodoxia y subalterniadad del flamenco como forma de comunicación cultural. Análisis del documental Petitet (Carles Bosch, 2018)
}

Nomadism, heterodoxia and subalternity of flamenco as a form of cultural communication. Analysis of the documentary Petitet

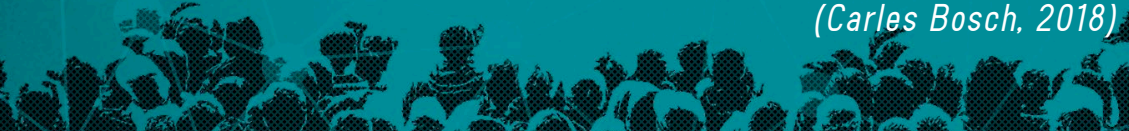

Miguel Alfonso Bouhaben

(E. Superior Politécnica del Litoral de Guayaquil, Ecuador)

[malfonso@espol.edu.ec]

DOI : http://dx.doi.org/10.12795/IC.2020.i17.07

E-ISSN: $2173-1071$

IC - Revista Científica de Información y Comunicación

2020, 17, pp. $143-166$

\section{Resumen}

En esta investigación abordaremos la problemática del deseo de las minorías gitanas de ser reconocidas por las élites culturales. Para ello, utilizaremos los conceptos de Gilles Deleuze y Félix Guattari para pensar el nomadismo del pueblo gitano en correlación con sus formas heterodoxas de comunicación cultural: el flamenco y la rumba catalana. Finalmente, sobre esta base teórica, analizaremos las formas de subalternidad cristalizadas en el documental Petitet (Carles Bosch, 2018)

\section{Abstract}

In this research, we will address the problem of the desire of gypsy minorities to be recognized by cultural elites. To do this, we will use the concepts of Gilles Deleuze and Félix Guattari to think about the nomadism of the gypsy people in correlation with their heterodox forms of cultural communication: flamenco and Catalan rumba. Finally, on this theoretical basis, we will analyze the forms of subalternity crystallized in the documentary Petitet (Carles Bosch, 2018)

Palabras clave

Pueblo gitano, flamenco, rumba catalana, comunicación cultural, subalternidad, Deleuze y Guattari.

\section{Keywords}

Gypsy people, flamenco, Catalan rumba, cultural communication, subalternity, Deleuze and Guattari. 


\section{Sumario}

1. Introducción

2. Metodología

3. Marco teórico

4. De la India a Cataluña. Nomadismos de la expresión musical del pueblo gitano

4.1. El nomadismo gitano: identidad desterritorializada e identidad heterogénea

4.2. El flamenco como forma de comunicación cultural heterodoxa

4.3. La rumba catalana como forma de comunicación cultural de ida y vuelta

5. De la Calle de la Cera al Liceo. Subalternidad lingüística y cultural en el documental Petitet (Carles Bosch, 2018)

5.1. Triglosia y lenguas menores en los gitanos catalanes

5.2. Subalternidad y multiculturalidad de la rumba catalana

6. Discusión y conclusiones

7. Bibliografía

\section{Summary}

1. Introduction

2. Methodology

3. Theoretical framework

4. From India to Catalonia. Nomadisms of the musical expression of the gypsy people

4.1. Gypsy nomadism: deterritorialized identity and heterogeneous identity

4.2. Flamenco as a heterodox form of cultural communication

4.3. Catalan rumba as a form of round-trip cultural communication

5. From Calle de la Cera to Liceo. Linguistic and cultural subordination in the documentary Petitet (Carles Bosch, 2018)

5.1. Triglossia and minor languages in the Catalan gypsies

5.2. Subalternity and multiculturalism of the Catalan rumba

6. Discussion and conclusions

7. Bibliography 


\section{Introducción}

En el documental Petitet (Carles Bosch, 2018) asistimos a la lucha titánica de un músico gitano de rumba catalana, por llevar las canciones más representativas de este subgénero musical al Gran Teatro Liceo de Barcelona. Este deseo del protagonista por vindicar la rumba catalana es en todo punto elogiable. Ahora bien, ¿por qué el protagonista, en tanto que sujeto de etnia gitana, minoritaria y subalternizada, desea el reconocimiento de su música en el espacio cultural de las élites dominantes? ¿por qué el flamenco, que es una forma de comunicación cultural de una riqueza extraordinaria, necesita del marco de sentido comunicacional y cultural de las élites para ser reconocida?

Esta pregunta, que moviliza la presente investigación, nos interpela a pensar porqué el flamenco -en general- y la rumba -en particular-, formas de comunicación cultural libres y heterogéneas, interiorizan de manera acrítica las formas de dominio cultural hegemónicas. Así, el objetivo de la investigación parte de la reflexión teórica en torno a dichas formas de comunicación cultural con el fin de abordar la problemática del deseo de las minorías gitanas de ser reconocidas por las élites culturales del capitalismo a partir del análisis del documental Petitet (Carles Bosch, 2018). De este modo, el documental será el material del estudio para valorar las formas de subalternidad lingüística y cultural del pueblo gitano catalán a las directrices normativas tanto de la máquina semiótica del catalanismo como del capitalismo. En suma, se trata de poner en crisis las formas de "exotización" del "Otro folclórico" y de apuntar a otras formas de expresión más libres y emancipadoras.

\section{Metodología}

Para dar cuenta de esta problemática, vamos a realizar dos viajes. En el primero, plantearemos la condición nomádica y desterritorializada del pueblo gitano y su construcción ontológica heterogénea como condición de posibilidad de la expresión comunicativaheterodoxa del flamenco y de la rumba catalana. Para ello, vamos a recurrir a una prolija variedad de conceptos forjados por Gilles Deleuze y Félix Guattari, en las décadas de los 70 y 80 , para proyectarlos sobre las problemáticas abiertas por el flamenco y la rumba catalana. Deleuze sostenía, en la conferencia “¿Qué es un creación?”, que en todos los campos de saber se trabaja con ideas, a pesar de su diferencias singulares -la ideas en filosofía son conceptos, las ideas en ciencia son funciones, las ideas en pintura 
son formas y colores, las ideas en música son bloques de melodía/armonía- y, por tanto, entre todas las disciplinas se pueden establecer nexos, filiaciones y encuentros. En nuestro caso, la idea era ver cómo las ideas musicales del flamenco y de la rumba podían dialogar y comprenderse a través de algunas ideas filosóficas de Deleuze y Guattari.Asimismo, en este primer viaje se harán uso de conceptos de estudios de gitanología y flamencología.

En el segundo viaje, describiremos las formas de subalternización del la rumba catalana a los contextos del catalanismo -a nivel lingüístico- y del capitalismo neoliberal -a nivel comunicativo y cultural a través del análisis del documental Petitet. Para ello, haremos uso de las teorías de la subalternidad y del multiculturalismo con el fin de pensar las formas de adaptación y sometimiento a las directrices culturales de las élites dominantes.

\section{Marco teórico}

De este modo, para desarrollar nuestro análisis de las formas de subalternización de las formas de comunicación cultural de la etnia gitanahemos acudido bibliografía específica sobre estudios deleuzianos, gitanología, estudios del flamenco, estudioslingüísticos, estudios subalternos y teorías críticas de la multiculturalidad.

Los conceptos de Deleuze y Guattari fueron los fundamentos teóricos que pusimos en práctica en los análisis sobre la etnia gitana así como sobre sus expresiones: el flamenco y la rumba. Las ideas de "mapa” "desterritorialización”, "rizoma”, "nomadismo", que Deleuze y Guattari elaboran en Mil Mesetas (1994) fueron aplicadas a los movimientos migratorios del pueblo gitano y a la configuración de su identidad; mientras que el concepto de "conexión heterogénea" fue relevante para entender la conformación heterodoxa de la expresión comunicativo-cultural del flamenco. De igual modo, el concepto de "menor", ensayado en Kafka, por una literatura menor (1978), la relaciones de poder lingüístico entre el catalán, el castellano y el romanés; mientras que los conceptos de "máquina", "código" y "flujo" forjados en Anti Edipo: capitalismo y esquizofrenia (2004) fueron de sumo provecho para pensar los mecanismos de normalización multicultural de las formas expresivas y comunicativas del flamenco.

Asimismo, fue necesario acudir a bibliografía sobre gitanología para pensar el origen del pueblo gitano (Gamella, Fernández, Nieto \& Adiego, 2011), su carácter nómada (Okeyly, 1983), las persecuciones sufridas (Paternina y Gamboa, 1999), sus procesos de inclusión sociocultural (Sordè, Flecha \& Mircea, 2013; Prieto i Flores, 2000) y su distribución a lo largo de la Península Ibérica (Sánchez Ortega, 1994; Gómez Urdáñez, 
2019). Además, tuvimos en consideración bibliografíasobre el origen gitano y andaluz del flamenco (Aguirre, 2006; Cruces Roldán, 2012; Grimaldos, 2010), sobre las músicas no ibéricas (Espejo, 2011; Goldbert, 2018; Nuñez 2008; Steingress, 2002) e ibéricas (Espejo, 2001; Leblon, 2005) que conforman el flamenco; sobre flamencología (Berlanga, 2012; Schuchardt, 1990), sobre el proceso heterodoxo del flamenco (Flor, 2011; Steingress 2004) y sobre rumba catalana (Castro, 2010; Castro, 2013; Fàbregas, 2006; Marfà, 2008),

Por último, para sostener y contrastar nuestra hipótesis tuvimos en cuenta estudios sobre lingüística de la lengua romanés (González, 2009; Saladrigues, 2000; Escudero, 2000), sobre triglosia (Ballón Aguirre, 2006; Fasla, 2006; Segre, 1979; Fishman, 1967), sobre estudios de la subalternidad (Modonesi, 2012 Mezzadra, 2008 Spivak, 2003), sobre la crítica al multiculturalismo (Žižek, 2008; Žižek, 1998) así como algunas ideas sobre la espectaculización del arte (Rancière, 2010) y sobre las diferencias entre alta y baja cultura (Pardo, 2007).

\section{De la India a Cataluña. Nomadismos de la expresión musical del pueblo gitano}

El primer viaje que vamos a trazar en el mapa es el que inician los gitanos desde la India hasta España. Sabemos que existen varias teorías sobre el origen de los gitanos pero, la más extendida, sin ánimo de instaurar un sesgo científico-ideológico, es aquella que aportan los estudios lingüísticos del romanés, que los sitúan en el Noroeste de la India:

Seguramente fue Johan Rüdiger, profesor en Halle, el primero en anunciar ese sensacional descubrimiento en abril de 1777, al publicar entonces un artículo que contenía el primer esbozo gramatical de un dialecto romanés $y$, en paralelo, comparaciones estructurales sistemáticas de ese lenguaje con el indostaní (Gamella, Fernández, Nieto \& Adiego, 2011: 4).

Para definir este primer viaje, será de utilidad el concepto de Deleuze y Guattari de mapa en tanto dispositivo "abierto, conectable en todas sus dimensiones, desmontable, alterable, susceptible de recibir constantemente modificaciones" (1994: 18). Al hilo de esta definición, pretendemos determinar tanto las formas de vida nómadas del pueblo 
gitano como las "conexiones" que establecen en su diálogo con otras formas culturales, que "alteran" y "modifican" su identidad. Y de ahí, estableceremos una relación entre estas formas nomádicas y las formas heterodoxas de comunicación musical: primero, con el flamenco; después, con la rumba catalana.

\subsection{El nomadismo gitano: identidad desterritorializada e identidad heterogénea}

Para abordar la identidad cultural del pueblo gitano, que si bien como toda determinación identitaria conlleva una inequívoca problematicidad histórica que puede desembocar en la cosificación y esencialización la identidad gitana,vamos a tener en cuenta dos aspectos.

En primer lugar, nos proponemos valorar la importancia del nomadismo en la configuración de las singularidades culturales del pueblo gitano. El éxodo que sufrieron determina una de sus características definitorias, a saber, la desvinculación de su identidad con el territorio. Esta vinculación identidad-territorio, propia de los Estados-Nación, es la condición de posibilidad de las formas de configuración política en un territorio delimitado por fronteras. La comunidad gitana, por el contrario, no posee un territorio y, por ende, podemos definirla -tomando prestada la terminología de Deleuze y Guattari (1994)- como un pueblo desterritorializado. Sin ánimo de consustancializar y esencializar al pueblo gitano, si resulta pertinente sostener que este carácter desterritorializado y viajero (Okeyly, 1983) se ha ido constituyendo a golpe de desigualdad, persecución y esclavitud, lo cual potencia su necesidad de movilidad, en su decurso histórico, así como sus interacciones con otras culturas: "La violencia y persecución sistemática de que han sido objeto los gitanos a través del tiempo por diferentes núcleos y en diversas sociedades ha contribuido a fortalecer en este grupo tribal sus prácticas itinerantes de vida" (Paternina y Gamboa, 1999: 158). En consecuencia, el nomadismo del pueblo gitano ha definido su singular modo de pensar, de vivir y de sentir: una forma anárquica y libertaria de ser en el mundo, una perspectiva que transciende la lógica de la historia, del sedentarismo y del Ser, para vindicar una práctica de vida en devenir. Frente a los Estados-Nación -con sus territorios, sus leyes, su razón- el pueblo gitano vive ajeno a las delimitaciones territoriales y apuesta por la nomadología "contra la forma-Estado" (Deleuze \& Guattari, 1994: 420). En suma, el nomadismo gitano forja una identidad ontológica desterritorializada. 
En segundo lugar, queremos hacer énfasis en cómo esta identidad ontológica desterritorializada es, a su vez, la condición de emergencia de una identidad cultural heterogénea. 0 lo que es lo mismo, esta identidad desterritorializada implica el contacto, el intercambio y la comunicación con otras culturas diversas -culturas con las que a veces se establece una relación de igualdad, horizontal y democrática; y otras veces, una relación asimétrica, cuando las culturas con las que establecen relación adoptan una posición de poder. Con todo, el pueblo gitano bascula entre la conservación de sus tradiciones y la apertura al diálogo comunicacional con las culturas contactadas, posibilitando así que sus diferencias sean incluidas en el seno de las culturas que atraviesa en su tránsito histórico. Así, la inclusión solo se puede dar en la dialéctica conservación/transformación: "la identidad gitana como aquella que debe permitir conservar la propia cultura y, al mismo tiempo, transformarla" (Sordè, Flecha \& Mircea, 2013: 6). De esta manera, la identidad gitana primero es deterritorializada y, después gracias a la intercomunicación cultural, deviene identidad heterogénea. En su proceso constructivo, la identidad gitana se define por su exterior, por las relaciones comunicativas con su afuera cultural: configura una identidad/multiplicidad que se transforma al conectarse con otras identidades: identidad metamórfica, identidad movediza, identidad en expansión, identidad alterable y identidad modificable. Como un rizoma (Deleuze \& Guattari, 1994).

Si bien es indudable el sentimiento de pertenencia nacional y el arraigo sedentario de muchos gitanos a lo largo del tiempo y en diversos contextos históricos, consideramos que la identidad, primero desterritorializada y después heterogénea, son la condición de existencia de su expresión artística definitoria, al menos en el ámbito ibérico: el flamenco.

\subsection{El flamenco como forma de comunicación cultural heterodoxa}

El flamenco sabemos que es el resultado de la expresión del pueblo gitano en su devenir nomádico desde la India -pasando por Irán y por Egipto- hasta llegar el sur de España. No obstante, para muchos teóricos, no es un arte exclusivamente gitano, sino que nace del diálogo y la comunicación intercultural gitano-andaluz. Con todo, sí que es cierto que fueron los gitanos de la Baja Andalucía los que preservaron oralmente la cultura del flamenco y luego la transmitieron al resto de la Península (Aguirre, 2006; Cruces Roldán, 2012; Grimaldos, 2010). 
Si bien parece consistente esta hipótesis, hay que establecer de manera más precisa de qué modo se constituye esta síntesis heterodoxa entre los elementos culturales de los migrantes gitanos y los elementos culturales autóctonos andaluces que, como hemos apuntado, posibilitan la emergencia de la compleja trama simbólica y comunicativa del flamenco. Para fundamentar esta síntesis, vamos a considerar dos niveles de interacción cultural.

El primer nivel se configura cuando los gitanos atraviesan medio mundo hasta llegar al sur de España. En ese devenir, los gitanos beben de las fuentes musicales melódicas, armónicas y rítmicas- de su transitar nómada por Oriente Medio y por el Norte de África. De modo que el flamenco, antes de configurarse, se va cociendo a través de la mezcolanza de sedimentos culturales que los gitanos van encontrando y recogiendo en su trayecto: por ejemplo, el quejío del flamenco conserva resonancias del cántico del almuédano cuando llama a la oración (Espejo, 2011: 26). Otros autores han introducido recientemente en sus investigaciones la importancia del elemento africano en la configuración del cante y baile flamenco (Goldbert, 2018; Nuñez 2008; Steingress, 2002).

El segundo nivel de interacción se da cuando los gitanos llegan a la Península Ibérica y sintetizan su heterogéneo legado musical con el legado autóctono. Como en el primer nivel, estas músicas autóctonas también componen un rico crisol de singularidades rítmicas, armónicas y melódicas fruto de la multiplicidad de culturas y pueblos que convivieron o batallaron a lo largo de siglos en la Península Ibérica. De ahí que el flamenco sea el resultado de las encrucijadas simbólicas de los diversos caracteres culturales de los gitanos y de los pobladores de la Baja Andalucía, esto es, la reelaboración, recreación y reinterpretación de las músicas autóctonas como las zarabandas, las gallardas y los romances; de las músicas hebreas de los judíos que vivieron en la Península y, sobre todo, de las formas de la música musulmana como la mohaxaja, la zambra o los zéjeles, en su interrelación con la cultura musical de los gitanos (Espejo, 2011: 26-28). Algunos teóricos de prestigio internacional como Bernard Leblon entienden que el flamenco es más bien producto de la síntesis de las músicas tradicionales autóctonas interpretadas de un modo singular de ejecución por los gitanos: "la música que tocan es la tradicional o popular de la tierra donde están, pero siempre con un “deje”, un sello característico" (2005: 109). Así, el flamenco solo es comprensible como un constructo heterogéneo de intersecciones y de intercomunicaciones de pluralidades culturales: de conversos, de moriscos, de cristianos y, por supuesto, de gitanos.

El carácter heterogéneo y mestizo -esto es, la conexión heterogénea (Deleuze \& Guattari, 1994) que está en el origen del flamenco- es argumento suficiente para poner 
en crisis el esgrimido por la ortodoxia del flamenco y, sobre todo, su precepto fundamental de la pureza. Para los ortodoxos, el flamenco es una suerte de "esencia pura": platónica, inalterable, universal e inmutable. De ahí que los ortodoxos se empecinen en tramar normas y reglas que sirvan para diferenciar qué es y qué no es flamenco. Y no solo reglas. Los flamencólogos impusieron unaeugenesia simbólico-cultural para definir a los flamencos puros, que desde su perspectiva debían ser cantaores gitanos de la Baja Andalucía: "Todo género asociado originariamente a los gitanos de la Baja Andalucía (seguiriyas, soleares, tonás, determinados romances) era auténticamente flamenco. En el cante gitano bajoandaluz estaba la raíz de las esencias [...] Es decir que el flamenco puro es el flamenco gitano" (Berlanga, 2012: 4). En la actualidad, después de los flamencólogos, también proliferan ortodoxos que pretenden guardar al flamenco en el tarro platónico de las esencias. Uno de los argumentos de los ortodoxos actuales radica en que defienden, con cierta razón, que la pervivencia del cante está en tela de juicio en esta posmodernidad globalizadora y neoliberal, donde todo se mezcla con todo, y donde los caracteres culturales y distintivos se difuminan.

Ahora bien, nuestra hipótesis apunta a la heterodoxia como la forma expresiva y comunicativa del flamenco. Hace más de un siglo, el filólogo Hugo Schuchardt criticaba la posición de los ortodoxos flamencólogos en su vindicación de la esencia gitana del flamenco: "Si los cantes flamencos proceden originariamente de una mezcla, ¿no es algo contradictorio decir que: "andaluzándose o haciéndose gachonales irán perdiendo poco a poco su primitivo carácter y originalidad y se convertirán en un género mixto?"” (Schuchardt, 1990: 20). El flamenco, como hemos apuntado, nace de una compleja hilazón comunicativa de multiplicidades culturales y, por tanto, podemos definirlo como un género musical mixto, una suerte de rizoma en el sentido deleuziano-guattariano. En el flamenco los caracteres musicales de las diversas culturas que lo atraviesan no establecen un ordenamiento racional, ni conforman un universal simbólico como pretenden los ortodoxos. Por el contrario, el flamenco es un cúmulo de dialectos musicales que se han ido aglutinando de forma libre y, por ello, las actuales comunicaciones del flamenco con el jazz, el rock o la bossa nova son parte consustancial de la lógica genética de flamenco. Ejemplos contemporáneos de mixtura del flamenco los podemos encontrar en el disco La leyenda del tiempo de Camarón de la Isla, donde por primera vez el flamenco utiliza bajo eléctrico; o en Omega, del cantaor Enrique Morente en diálogo con los ruidosos rockeros de Lajartija Nick. En ambos casos, encontramos una crítica al esencialismo del flamenco: una crítica desde una praxis nomádica, rebelde y heterodoxa, que ha tenido que batallar contra la intolerancia irracional de los ortodoxos alegando, como Camarón, que "lo que tiene que 
hacer la gente es oírme más, hasta comprenderme"; o afirmando, como Morente, que "se habla de los cambios en el flamenco con mucha vigilancia, pero esta música ha estado modificándose constantemente desde sus orígenes".

Por tanto, podemos afirmar que el flamenco forja descentramientos y deconstrucciones de las músicas existentes, funcionando como una auténtica máquina descompositivo/recompositiva musical que no hace otra cosa que expresar y comunicar los procesos desterritorializados y heterogéneos del pueblo gitano. Si el pueblo gitano es un pueblo en devenir, un pueblo desterritorializado y nómada que dialoga y se intersecta con otras culturas, entonces, sus expresiones artísticas, como ocurre con el flamenco, tendrán ese mismo carácter dinámico, abierto y heterogéneo. Podemos entender el flamenco como una música movediza y plástica que tiene un gran potencial para mezclarse, comunicarse y articularse con otras músicas: "como una gran esponja que absorbe todo, pero nunca pierde su forma” (Flor, 2011: 11). Para nosotros, más bien, esa forma es siempre una forma in progress, una forma heterodoxa, una inter-forma que surge de la intercomunicación cultural. Por ello, compartimos con Gerhard Steingress la idea de que "el flamenco andaluz es un ejemplo idóneo para demostrar un proceso de hibridación transcultural” (2004: 3).

\subsection{La rumba catalana como forma de comunicación cultural de ida y vuelta}

Tras su llegada a Andalucía, los gitanos se distribuyeron progresivamente por toda la Península Ibérica (Sánchez Ortega, 1994; Gómez Urdáñez, 2019). Según fuentes de la Fundación Secretariado General Gitano de España, el 46\% de los gitanos vive en Andalucía; el 13\% lo hacen en Cataluña; y el 10\% en Comunidad Valenciana y en Comunidad de Madrid, respectivamente. Esta distribución va a dar lugar a nuevas formas musicales ya que, en cada uno de esos lugares, surgen nuevos encuentros que posibilitan nuevos cantes, como es el caso de la rumba catalana: un cante de ida y vuelta $-y$, por tanto, de comunicación cultural heterodoxa- que tiene su arraigo geográfico en la ciudad de Barcelona.

La rumba catalana guarda similitudes con la rumba flamenca y, por ello, podemos considerarla un palo del flamenco. Como aquella, también posee un carácter fuertemente heterodoxo: manifiesta los procesos de hibridación y transculturación inherentes al flamenco dada su condición de cante de ida y vuelta. Apuntemos que los cantes de ida y vuelta son formas híbridas de comunicación cultural que beben de fuentes hispanoamericanas que, a su vez, tienen su origen atávico en músicas de origen español. 
La base fundamental de la rumba es el son cubano, que surgió de la comunicación de estilos musicales africanos con músicas canario/andaluzas. Tras este viaje de ida de las músicas de origen español, que fundan junto con ritmos afro la rumba cubana, comienza el viaje de vuelta. Son muchos los investigadores que defienden esta hipótesis. Blas Vega, por ejemplo, sostiene que "de todos los estilos de influencia hispanoamericana es la rumba la que en la actualidad conserva su vigencia, pues hacia 1957 empieza a ponerse de moda la rumba catalana, adaptación de los gitanos de Barcelona, en la que predomina cierto ritmo o carácter flamenco" (en Castro, 2010: 167). Por su parte, el guitarrista Andrés Batista escribe:

de origen afro-cubano, con aire alegre y cadencioso, la Rumba está incluida por los flamencólogos en los estilos considerados de Ida y Vuelta. Muchos artistas flamencos la interpretan, sobre todo para el baile; pero son los kalós catalanes quienes ponen de moda este estilo, por su peculiar aire rítmico y forma de interpretación (en Castro, 2010: 168).

Ahora bien, este carácter heterogéneo de la rumba catalana hace que tanto los ortodoxos del flamenco como los ortodoxos de la rumba cubana renieguen de ella o la consideren un género menor, seguramente porque la perciben como una forma de comunicación cultural mestiza, incómoda y resistente.

Así, la rumba es una música en devenir que emerge del diálogo comunicativo con las músicas-otras con las que se va encontrando en el camino. Si el flamenco es el resultado de la intersección entre lo gitano y lo andaluz; la rumba catalana es un palo del flamenco igualmente intersticial, que comunica lo gitano y lo catalán, con una localización icónica: la calle de la Cera en Barcelona -sin olvidar que el barrio de Gracia y el de Hostafrancs-Badal son también dos de los centros originarios de la rumba catalana (Castro, 2013; Fàbregas, 2006)- lugar donde habitaban los gitanos catalanes que son considerados los depositarios de esta tradición flamenca. Cabe apuntar que la doble identidad gitano-catalán les moviliza a diferenciarse tanto de los catalanes payos como de los gitanos no catalanes. De este modo, los gitanos catalanes se mueven en ese espacio híbrido entre la tradición y la asimilación, la cual, les permite la “interacción con la sociedad paya mayoritaria, no sólo porque en la producción discográfica y la interpretación musical participan payos, sino porque los formatos de canción pop que inaugura Peret tienen un claro propósito de llegar al gran público" (Marfà, 2008: 61). De este modo, estos procesos 
de asimilación les llevan a priorizar los elementos culturales del catalanismo. Como señala María Jesús Castro, la rumba catalana es "equivalente a "gitano catalán" pero, sobretodo, de "catalán", con una determinada cultura diferenciada de los payos no-catalanes y de los kalós no-catalanes, de esta forma los kalós utilizan la "rumba catalana" para reivindicar una catalanidad esencialista" (2010: 176).

Si bien es cierto que en Cataluña la identidad gitana tiene una construcción panétnica (Prieto i Flores, 2000) y que no hay una esencia gitano-catalana definida al extremo; es preciso señalar que entre el carácter comunicativo-cultural heterodoxo de la rumba -en tanto cante de ida y vuelta, música desterritorializada, heterogénea y nomádicay la posición catalanista de los gitanos catalanes, se establece una profunda tensión cultural que es preciso desvelar.

\section{De la Calle de la Cera al Liceo. Subalternidad lingüística y cultural en el documental Petitet (Carles Bosch, 2018)}

Una vez trazado el viaje que lleva desde el Noroeste de la India, de donde son originarios los romaníes, en un periplo que va nutriendo y enriqueciendo sus expresiones musicales, en una suerte de exploración y de experimentación por la cartografía del planeta, abierto a los contactos, las comunicaciones y las conexiones con otras culturas, alterando en cada paso su identidad cultural, hasta Cataluña; vamos a dibujar otro viaje, más inquietante desde cierto ángulos, un viaje hacia la pérdida de la razón nomádica, libre y ácrata del pueblo gitano: el corto viaje que va de la Calle de la Cera al Teatro Gran Liceo de Barcelona.

Para dar cuenta de este extraño viaje es necesario relatar, siquiera brevemente, el argumento de la película documental Petitet, cuyo visionado fue el detonante de la presente investigación. Ahora bien, antes de empezar, es preciso indicar que, en lo que sigue, vamos a tomar como objeto de análisis los elementos lingüísticos y culturales que aparecen cristalizados en el documentalPetitet y, por ello, no se valorará la perspectiva crítica que el músico pueda adoptar frente al documental. Dicho esto, el argumento del film nos presenta a Joan Ximénez, conocido como Petitet, un gitano del barrio barcelonés del Raval que antaño fue un percusionista que acompañó a grandes músicos de la rumba catalana. É mismo cuenta que su padre fue palmero del gran ídolo Peret. Al inicio de la película, Petitet acude con su hija al cementerio para poner flores a su madre. En ese momento, el protagonista 
confiesa que le prometió llevar la rumba catalana al Gran Teatro del Liceo: "Para un gitano la promesa a una madre lo es todo". Esa promesa, justamente, es la que moviliza el deseo del protagonista y la que desata la epopeya del héroe por conformar un equipo de músicos para conseguir la hazaña prometida. Se van sucediendo las complejidades en los ensayos y las discusiones -la mayoría son músicos no profesionales con un gran talento, pero con falta de disciplina- que van configurando las tensiones del relato. Un relato donde vindica una posición ortodoxa respecto a la rumba: "Yo no voy a inventar nada. Ya está todo inventado". En paralelo a los ensayos, el protagonista va desgranando las particularidades de la calle de la Cera, el lugar donde nació la rumba catalana, que está situada justo detrás del Liceo.

Ahora bien, ¿qué implicaciones políticas y culturales tiene este viaje de la calle de la Cera al Liceo? ¿Cuál es el peaje a pagar en el trayecto? Anticipamos que éste es doble: implica la pérdida de la lengua, la minusvaloración radical llegando casi a la desaparición de la lengua romanés; así como la pérdida de la cultura, al adoptar una posición subalterna al visibilizar el deseo de ser comprendido y aceptado en los espacios de las élites de la cultura dominante. Lo paradójico radica en que el esfuerzo de Petitet por reivindicar la rumba como una forma de comunicación cultural de la que sentirse orgulloso le lleva, inconscientemente, a despreciarla de dos modos: primero, a través del abandono de su lengua originaria -el romanés- para situarla en un status sociocultural menor en favor de la lengua dominante catalana; segundo, abandonandoprogresivamente los elementos singulares y diferenciales de sus formas de comunicación cultural para satisfacer el deseo y el gusto de multiculturalidad del capitalismo neoliberal globalizado.

\subsection{Triglosia y lenguas menores en los gitanos catalanes}

A través de Petitet se expresa un panfleto político de raigambre catalanista, esto es, un alegato en pro los valores, las tradiciones, la cultura y la lengua catalanas. Es interesante constatar como el catalanismo, que aglutina tendencias políticas de todo signo, así como a ciudadanos con arraigo de generaciones, junto con otros charnegos y recién Ilegados; ha calado hondo entre los gitanos. Esta alineación con las reivindicaciones del catalanismo les lleva a los gitanos a poner en mayor valía la cultura catalana en detrimento de las tradiciones gitanas, que van perdiendo peso específico. Sobre todo a nivel lingüístico, ya que el documental nos muestra toda una jerarquía de los diferentes usos comunicativos de las lenguas catalana, castellana y romanés o caló. Esta última, sumida en el más absoluto de los silenciamientos. 
Respecto a las relación jerárquica entre la lengua catalana y la castellana en el documental, hay que señalar una diferencia ineludible: por un lado, se observa como los gitanos rumberos, cuando se comunican entre ellos o cuando hablan a cámara, casi todos ellos lo hacen en catalán: mientras que las canciones que preparan para su concierto en el Liceo, todas ellas, están escritas en castellano. Al inicio de la película se subraya esta pauta dialéctica que surca todo el film: en la secuencia del cementerio, de fondo, suena Una lágrima cayó en la arena de Peret, padre de la rumba catalana. Poco después, escuchamos al propio Petitet hablar sobre sus padres en catalán: "Era tot. La familia es tot". Tenemos que esperar hasta el minuto cinco para escuchar al primer gitano -uno de los pocos que lo hace- hablar en castellano. Después, encontramos otros momentos donde se habla en castellano: en la secuencia donde aparece el primer cantante -al cual Petitet le regaña por no ser responsable y llegar con resaca a la grabación- y en la secuencia donde aparece el segundo cantante, el Granaíno. Tenemos que esperar hasta el minuto treinta y cuatro para poder escuchar a Petitet comunicarse en castellano. Es en la secuencia donde habla con su hija.

Una vez que hemos visibilizado la diferencia cuantitativa y cualitativa en los usos del catalán -para la comunicación social- y del castellano -para las canciones-, nos queda ver el lugar que ocupa la lengua originaria de los gitanos. Hay que señalar que el romanés o caló es un "pogadolecto que ha tomado la estructura gramatical del español y sobre ella ha ido insertando el vocabulario romanó" (González, 152: 2009). Por ello, parece lógico que si el castellano va perdiendo fuerza en Cataluña, al menos en las instituciones públicas, entonces el romanés, que está tradicionalmente adherido al castellano, también va a perder protagonismo. En este sentido, no resulta contradictorio que durante toda la película no aparezca ninguna de las palabras del romanés, palabras que en otras latitudes de España los gitanos utilizan con asiduidad, palabras que incluso han pasado tanto al habla común como al Diccionario de la RAE. Ni siquiera en momentos de intimidad, como cuando habla sobre la familia, Petitet o sus familiares utilizan vocabulario. Aunque, todo hay que decirlo, esta intimidad es más bien presunta y artificiosa, ya que en todo momento el protagonista es consciente de la presencia de la cámara.

En esta compleja relación entre el catalán, el castellano y el romanés o caló se establecen vínculos jerárquicos que nos convocan a defender la existencia de triglosia entre los gitanos catalanes. Hablamos de triglosia y no de trilinguismo cuando se establece una relación de dominio comunicacional cultural entre tres lenguas que conviven en un mismo territorio. Hay multitud de estudios que han abordado la triglosia en otras latitudes -en Bolivia, donde conviven de forma asimétrica el quechua, el castellano y el aimara 
(Ballón Aguirre, 2006) o en Marruecos, donde lo hacen el árabe, el bereber y el francés (Fasla, 2006)-; y en otros momentos de la historia -como el trabajo de Cesare Segre (1979), "La tradizione macaronica da Folengo a Gadda (e oltre)", donde estudia la relación entre el latín, el toscano y el dialecto local en la Italia de 1300.

Por otro lado, es importante apuntar que si la diglosia hace alusión a una relación de poder entre dos variedades lingüísticas, donde una tiene un papel preferencial -ya sea porque es la de uso oficial o la que mas prestigio cultural aporta- y donde otra adopta un papel subalterno -relegada a la vida familiar 0 al folckore-; en la triglosia se establecen jerarquías entre tres variedades linguísticas. Fishman (1967), uno de los teóricos más prestigios de la triglosia, considera que hay que tener en cuenta dos factores fundamentales: 1) las lenguas deben de tener un origen diferente $y, 2$ ) las lenguas deben de tener un status social dispar. Si bien el catalán y el castellano comparten origen romance, entre ellos se establece una distinción de status social. En el caso que nos ocupa, la relación de dominio comunicativo entre las tres lenguas de los gitanos catalanes -queremos subrayar que nos referimos a la relación de dominio en el documental y no en la realidad social- resulta sumamente significativa. El catalán es, indiscutiblemente, la variedad lingüística dominante: casi todos los gitanos del documental se comunican en catalán, solo hay un gitano que lo hace en castellano. Sin duda, el catalán es la lengua oficial de los gitanos catalanes, la variedad alta y prestigiosa, que les permite asimilar y adaptarse a la cultura dominante catalana. Es la variedad aprendida en un contexto social que se ha vuelto hegemónica, ya que en las comunidades gitanas actuales del barrio del Raval o de Gràcia hablan entre sí exclusivamente en catalán (Saladrigues, 2000). El castellano, por su parte, es una variedad comunicativa media. El protagonista, como hemos apuntado, lo habla en situaciones familiares -véase la secuencia con su hija.Igualmente, el castellano es utilizado en sus relaciones comunicativas con otros gitanos o payos no-catalanes. El caló o romanés, por último, que ha desaparecido completamente en el film, es una variedad baja ya que fue abandonada al adoptar las lenguas vehiculares (Escudero, 2000).

Al hilo de estas complejas relaciones de poder entre las lenguas, resultan pertinentes las consideraciones de Deleuze y Guattari en su obra conjunta Kafka, por una literatura menor. La praxis lingüística de los gitanos catalanes del documental está atravesada un concepto que ambos filósofos ensayaron en esta la obra, el concepto de menor: "Una literatura menor no es una literatura de un idioma menor, sino la literatura que una minoría hace dentro de una lengua mayor" (28: 1978). Aplicando esta idea a los gitanos de Petitet, podemos advertir tres niveles de relaciones jerárquicas en la lengua: 1) La lengua menor de los gitanos, el caló o romanés, que si bien no aparece en 
la película sí que es parte integral de su música, resultado de una tradición milenaria, con una multiplicidad de contactos y devenires; 2) La lengua intermedia de los gitanos ejemplificada en las letras de las canciones interpretadas en castellano; 3) La lengua mayor, lengua del contexto social de la lengua dominante: el catalán. La relación jerárquica entre las lenguas implica una tensión entre la constante -la dominante- y la variación -la menor-, como sostienen ambos años después en Mil mesetas(1994: 105).

En suma, se observa una "relación triglósica" de subordinación lingüística vinculada a la estructura sociopolítica, así como una exposición de las minorías de la lengua, donde se ve claramente que el catalán es la variedad lingüística de uso social dominante, la que sirve para comunicarse; el castellano es de uso popular, de ahí que prácticamente solo se use en las canciones; y el caló, es la variedad que está sumida en el olvido y silenciada.

\subsection{Subalternidad y multiculturalidad de la rumba catalana}

Una vez descrita la posición menor de la lengua romanés con respecto al castellano y al catalán, vamos a tratar de describir, a través del film, la posición cultural subalterna que adoptan los gitanos respecto a la cultura dominante. A pesar de que parte del colectivo gitano no forma parte de un colectivo marginado -de hecho en muchos gitanos catalanes se da un alto nivel de integración sociocultural- no deja de ser cierto que sus modos vida están regulados no solo por la cultura catalana, como ya vimos en el territorio de la lengua, sino que también por los férreos cimientos de la cultura del capitalismo neoliberal de corte internacionalista. Para vislumbrar el funcionamiento de la cultura del capitalismo dominante es preciso acudir a dos conceptos co-implicados, el concepto de subalternidad y el concepto de multiculturalidad, de cara a observar su intersección en nuestro estudio de caso.

Vamos a estimar como el concepto de subalternidad nos puede servir para pensar dos formas de subalternidad en Petitet. La primera, está marcada por la relación de dominación y negación histórica -en este caso del pueblo gitano- que posibilita la construcción de la subjetividad subalterna que vamos a denominar "subalternidad ontológica". La segunda, queda definida por la negación a tomar la palabra y hace referencia a la forma de la subalternidad que sustrae el logos -razón y palabra- y la episteme -saber- y, por ello, la vamos a definir como "subalternidad epistémica".

De acuerdo con esto, queda patente que el deseo de Petitet de llevar la rumba 
catalana de la calle de la Cera al Gran Teatro del Liceo responde a una estructura de dominación: estructura donde su etnia ocupa una posición subordinada histórica a pesar de la integración sociocultural. Siguiendo a Marx, Antonio Gramsci define a los dominados como subalternos: "Io subalterno como expresión de la experiencia y la condición subjetiva del subordinado, determinada por una relación de dominación" (en Modonesi, 2012: 3).Para el pensador italiano, el concepto de subalterno está articulado con el concepto de hegemonía: el subalterno incorpora la opresión hegemónica, pero al mismo tiempo visibiliza su resistencia. Así, teniendo en cuenta la primera forma de subalternidad que hemos definido, cabe señalar que la condición subjetiva de Petitet está definida por una relación de dominación y, justamente por eso, desea ser aceptado por esas mismas estructuras que le oprimen. Es decir, el protagonista incorpora la dominación, la incrusta en el interior de su carne: aloja dentro de sí la negación histórica de sus superiores. Estas formulaciones gramscianas serán de los pilares teóricos de los Subaltern Studies, conformada por pensadores hindús en los años 80 , con el fin de revelar las voces negadas de los campesinos de la India. Ranajit Guha, el fundador de la Escuela, escribe acerca del campesino indio que su identidad "consistía en la magnitud de su subalternidad. En otros términos, aprendía a reconocerse no a través de las propiedades y atributos de su propia existencia social, sino desde una reducción, cuando no una negación, de los de sus superiores" (en Mezzadra, 2008: 26). Este reconocimiento ontológico del subalterno gracias a sus superiores resuena en el documentalen la medida que lo que se clava en el interior del sujeto es su condición racial, a saber, la inferiorización histórica proyectada sobre la etnia gitana. De ahí que el deseo del oprimido -justo porque incorpora la inferiorización y hace de ella un lastre- es salir de su raza. Ahora bien, al no poder renegar de su condición racial, por ejemplo en una suerte de mutación blanqueante, decide sustituir su condición racial por su condición de clase para renegar así de esta y optar por ascender socialmente. Ese deseo de ascenso social es el que late detrás del ideal de actuar en el Liceo. Recordemos que la calle de la Cera está justo detrás del Liceo. Es algo así como el patio de atrás: el inconsciente de clase que está invisibilizado esperando acceder al consciente del Liceo, de la cultura dominante.

La segunda forma, la "subalternidad epistémica", la podemos observar en la secuencia donde el guitarrista, catalán payo, demuestra sus conocimientos musicales cuando le preguntan por el nombre de un acorde y contesta: "Fa sostenido semidisminuido". En ese momento, se visilibiliza la subalternidad epistémica y todos ellos parecen rendirse ante la presunta superioridad de los saberes dominantes occidentales. Si bien es verdad que el pueblo gitano es un pueblo orgulloso de su cultura oral, la escena deja una extraña 
sensación, pues ríen durante un breve rato y dejan de hablar. Y no hablan, como apunta Spivak, porque no tienen historia:"no hay sujeto subalterno que pueda conocer y hablar por sí mismo (...) el subalterno no tiene historia y no puede hablar" (2003: 328). Es decir, no tienen una historia reconocida por la cultura dominante. Toda la riqueza histórica, oral y cultural de los gitanos es infravalorada, silenciada y negada en ese "Fa sostenido semidisminuido".

En este punto, es necesario señalar la tensión que se establece entre las nociones de multiculturalidad y subalternidad. Si bien es cierto que el proceso constitutivo de la música flamenca es netamente multicultural, esto es, se funda gracias de una serie de comunicaciones e hibridaciones melódicas, armónicas y rítmicas que se establecen en un mismo plano de igualdad, en una horizontalidad democrática y según la lógica del diálogo de saberes; la relación que establece la rumba catalana -al menos desde la perspectiva actual que plantea el documental- con otras expresiones culturales es de subalternidad.

Por ello, vamos a ver de qué modo se resuelve la tensión conceptual entre la multiculturalidad y la subalternidad. Sin duda, la cultura dominante esta ávida de novedades pintorescas y exóticas, de ahí que todos los espacios de las élites culturales -museos, cines, teatros- estén deseosos de dar cabida a productos de las otredades culturales. Esas otredades no son más que mercancías y, por ello, todo aquello que tengan de auténtico, resistente o crítico es desactivado dentro del marco mercantil. Recordemos a Jacques Rancière (2010) que sostenía que cuando el arte de protesta se espectaculariza, entonces termina por ser cómplice de sus presuntos enemigos. De igual modo, cuando las expresiones exóticas de la minoría gitana -en tanto que expresión desviada o no hegemónica- se espectacularizan, siempre según el marco de sentido de la cultura dominante, entonces estas pasan al lado de la hegemonía, eso sí, pagando el siniestro peaje de ponerse del lado de esa misma estructura de dominio que les subalterniza. De este modo, podemos afirmar que la tensión multiculturalidad/subalternidad se resuelve según la lógica multicultural del capitalismo. En este punto, compartimos el planteamiento de Žižek:

¿Y si la forma habitual en que se manifiesta la tolerancia multicultural no fuese, en última instancia, tan inocente como se nos quiere hacer creer, por cuanto, tácitamente, acepta la despolitización de la economía? Esta forma hegemónica del multiculturalismo se basa en la tesis de que vivimos en un universo post-ideológico (...) en el 
que las batallas más importantes serían aquellas que se libran por conseguir el reconocimiento de los diversos estilos de vida. Pero, ¿y si este multiculturalismo despolitizado fuese precisamente la ideología del actual capitalismo global? (Žižek, 2008: 11)

La lectura de Žižek del multiculturalismo resulta de utilidad para interpretar ese deseo del subalterno -del gitano catalán- de ser reconocido en los espacios de comunicación cultural del capitalismo -el Liceo. En eso consiste la tolerancia multicultural del capitalismo: la praxis del reconocimiento de los "diversos estilos de vida" siempre dentro del marco de sentido dominante del capitalismo. Una tolerancia que devela el núcleo del racismo contemporáneo en tanto que reconocimiento del Otro folclórico: "La "tolerancia" liberal excusa al Otro folclórico; privado de su sustancia (como la multiplicidad de "comidas étnicas" en una megalópolis contemporánea), pero denuncia a cualquier Otro “real” por su "fundamentalismo"”. (Žižek, 1998:157). En nuestro estudio de caso, se tolera la esencia exótica de las formas de comunicación cultural de la rumba catalana siempre y cuando se ajuste al marco del Otro folclórico, esto es, siempre y cuando la rumba sea susceptible de ser normalizada.

Este dispositivo de normalización del multiculturalismo en tanto que aparato ideológico del capitalismo funciona como una máquina de reorganizar, codificar y resignificar todos los flujos que escapan de su lógica (Deleuze \& Guattari, 2004). La rumba catalana podemos identificarla como un flujo deterritorializado que escapa de la lógica totalitaria cultural del capitalismo y, por ello, las industrias culturales del capitalismo, ya desde los años 50 y 60,se encargan de crear los dispositivos de normalización mercantil adecuados a ella. Son estos los que construyen la subjetividad subalterna del gitano, que desea que la rumba catalana sea reconocida. Es más útil reterritorializar el flamenco o la rumba catalana que marginarlo. Por ello, en el contexto posmoderno, ya no importa la vieja división entre la alta cultura, de carácter de difícil, complejo y determinada por el sentido de ascensión; y la baja cultura, de carácter fácil y gratuito y definida por el sinsentido y la pérdida de tiempo (Pardo, 2007). De algún modo, el flamenco -en general- y la rumba catalana -en particular- tenían antaño una esencia festiva y gratuita, ya que se tocaba y cantaba por puro placer, sin objetivos ni pretensiones. Pero cuando el flamenco y la rumba se convierten en negocio, entonces se introduce ese deseo de sentido y de ascensión de clase. Eso es, justamente, lo que le ocurre a Petitet. Quiere dar sentido a la rumba, sacarla del sinsentido de la fiesta y posibilitar que ascienda socialmente al presentarla en el Liceo. 


\section{Discusión y conclusiones}

Estos dos viajes que hemos dibujado -con el fin de poner en valor el viaje de ida y vuelta que constituye la rumba catalana- son en todo punto diferentes. En el primer viaje, hemos abordado fundamentalmente el carácter nomádico del pueblo gitano y, por ello, su condición libre y anárquica, al encuentro de otras culturas con las que intersectarse. De estas intersecciones, nace la posibilidad del flamenco, en tanto composición heterogénea de expresiones culturales en apertura, libertad, alteración, improvisación. En el segundo viaje, sin embargo, el movimiento es diferente. Es un viaje a la normalidad lingüística del catalanismo y a la normalidad multicultural del capitalismo contemporáneo. Llevar la rumba catalana de la calle de la Cera al Liceo, supone la pérdida de la batalla por la autonomía cultural.

Ahora bien, es sobre todo en los espacios culturales del capitalismo donde se ejerce la "exotización" del Otro folclórico, no con la intención de aportar un empuje a su emancipación, sino con una finalidad normativa y reguladora. En los Liceos, el menor siempre pierde. Es imposible para él ganar. Estos espacios, igual que los museos o los teatros o los cines, son auténticos "panópticos de la expresión”(Bouhaben, 2019: 254). Si el pueblo gitano es nómada y sus expresiones culturales son heterogéneas, resulta inquietante que la rumba, que nace del diálogo y la síntesis entre músicas iguales afro, cubanas, andaluzas, catalanas- se doblegue y someta a la cultura del capitalismo multicultural. La esencia y el color de la rumba se tiene que transformar y adecuarse al gusto de las élites, esto es, debe de ser sinfónica, para poder acceder al Liceo. Ese deseo, engrana con el problema de la política según Spinoza replanteado por Wilhelm Reich: “¿Por qué combaten los hombres por su servidumbre como si se tratase de su salvación?" (en Deleuze \& Guattari, 2004).

Ante este panorama, el viaje puede ser una buena metáfora para desactivar los dispositivos codificadores del capitalismo multicultural. Viajar es "partir, evadirse, es trazar una línea [...] Huir es trazar una línea, líneas, toda una cartografía. Solo hay una manera de descubrir mundos: a través de una larga fuga quebrada. El devenir es geográfico" (Deleuze \& Parnet, 1980: 45-46). Y eso son, en definitiva, el flamenco y la rumba catalana: un viaje desterritorializante, un devenir, una línea de fuga. Y eso es lo que más teme el multiculturalismo neoliberal: la improvisación, lo imprevisible, lo indeterminado, el viaje.

Un viaje a ninguna parte. 


\section{Bibliografía}

Aguirre Felipe, J. (2006). Historia de las itinerancias gitanas. De la India a Andalucía. Zaragoza: Institución "Fernando el Católico".

Ballón Aguirre, E. B. (2006). Tradición oral peruana: literaturas ancestrales y populares (Vol. 2). Lima: Fondo Editorial PUCP.

Berlanga, M. A. (2012). Repensando el flamenco en claves musicales. Líneas de investigación. En Olarte Martínez, Matilde (Ed.) Fuentes documentales interdisciplinares para el estudio del Patrimonio y la Oralidad en España, ed.. Pontevedra: Ed. Dos Acordes.

Bouhaben, M. A. (2019). Continuidades y discontinuidades estético-políticas del vídeo expandido. Análisis de mudanzas de María Teresa Ponce. Chasqui , 140.

Castro M. J. (2010). La rumba catalana y el flamenco como marcadores culturales de los kalós catalanes en Barcelona. Barcelona: Universitat de Barcelona.

Cruces Roldán, C. (2012). El flamenco. En Isidoro Moreno y Juan Agudo (Eds.) Expresiones culturales andaluzas. Sevilla: Aconcagua Libros

Deleuze, G., y Guattari, F. (1978). Kafka, por una literatura menor. México: Era.

--- (1994). Mil mesetas. Capitalismo y esquizofrenia. Valencia: Pre-textos.

--- (2004). El Anti Edipo: capitalismo y esquizofrenia. Barcelona: Paidós Ibérica.

Deleuze, G., y Parnet, C. (1980). Diálogos. Valencia: Pre-textos.

Escudero, J-P. (2000). Algunes dades sobre els gitanos de Catalunya i la seva antiga Ilengua.Treballs sociolingüística catalana 14 (15), 53-58. https://publicacions. iec.cat/repository/pdf/00000052/00000092.pdf

Espejo, J. A. (2011). Las leyes y el flamenco. Revista de Investigación sobre Flamenco La Madrugá (4), 21-36. https://revistas.um.es/flamenco/article/view/132211 
Fàbregas, B. (2006). La rumba catalana: panoràmica etno-històrica i recerca arxivística i documental dels seus origens. Barcelona: Centre de Promoció de la Cultura Popular i Tradicional Catalana, 2006.

Fasla, D. (2006). La situación lingüística del Magreb: lenguas en contacto, diglosia e identidad cultural. Revista española de lingüística (36), 157-188. https:// dialnet.unirioja.es $/$ servlet/articulo? codigo $=2563067$

Fishman, J. (1967). Bilingualism with and without diglossia, diglossia with and without bilingualism. Journal of Social Issues (2), 29-38. https://spssi. onlinelibrary.wiley.com/doi/abs/10.1111/j.1540-4560.1967.tb00573.x

Flor, C. de (2011). Flamenco. Madrid: Difusión, Centro de Investigación y Publicaciones de Idiomas, S.L.

Gamella, J. F., Fernández, C., Nieto, M., y Adiego, I. X. (2011). La agonía de una lengua. Lo que queda del caló en el habla de los gitanos. Parte I. Métodos, fuentes y resultados generales. Gaceta de Antropología ( 27), 1-31.

http://www.ugr.es/ pwlac/G27_39Juan_Gamella-y-otros.html

Gómez Urdáñez, J. L. (2019). Lo que pensaban sobre los gitanos los gobernantes del siglo ilustrado/What the rulers of the Enlightenment thought about the gypsies. Vegueta: Anuario de la Facultad de Geografía e Historia, 19: 535-551. http://www.ugr.es/ pwlac/G27_39Juan_Gamella-y-otros.html

Goldbert, K. Meira. (2018).Sonidos Negros: On the Blackness of Flamenco (currents in Latin American and Iberian Music).0xford: Oxford University Press.

González, N. J. (2009). ¿El romanó, el caló, el romanó-kaló o el gitañol? Cincuenta y tres notas sociolingüísticas en torno a los gitanos españoles. Anales de Historia Contemporánea (25), 149-161. https://dialnet.unirioja.es/servlet/ articulo? codigo $=2922497$

Grimaldos, A. (2010). Historia social del flamenco. Barcelona: Península.

Leblon, B. (2005). Gitanos y Flamenco. Valencia: Asociación de Enseñantes con Gitanos.

Machado Álvarez, A. (1881). Colección de Cantes Flamencos recogidos y anotados por Demófilo. Sevilla: Imprenta y Litografía de El Porvenir. 
Marfà, M. (2008). ¿Muerta o de parranda?: auge, caída y nuevo esplandor de la rumba catalana. Minerva: Revista del Círculo de Bellas Artes (8), 55-61. https:// www.circulobellasartes.com/revistaminerva/articulo.php?id=252

Mezzadra, S. (2008). Introducción. En Sandro Mezzadra (Ed.) Estudios postcoloniales: ensayos fundamentales. Madrid: Traficantes de sueños.

Modonesi, M. (2012). Subalternidad. México: UNAM.

Núñez, F. (2008).Guía comentada de cantos y bailes preflamencos (1750-1808). Barcelona: Ediciones Carena.

Okely, J. (1983).The traveller-Gypsies. Cambridge: Cambridge University Press.

Pardo, J. L. (2007). Esto no es música. Introducción al malestar en la cultura de las masas. Barcelona: Galaxia Gutenberg.

Paternina, H.A., y Gamboa, J. C. (1999). Los gitanos: tras la huella de un pueblo nómade. Nómadas, 10: 156-170. https://www.redalyc.org/pdf/1051/105114274013.pdf

Prieto i Flores, Ó. (2007).Sobre la identidad gitana y su construcción panétnica: el caso gitano en Barcelona. Barcelona: Universitat de Barcelona.

Rancière, J. (2010). El espectador emancipado. Buenos Aires: Ediciones Manantial.

Saladrigues, E. (2000). Aspectes lingüístics i culturals dels gitanos de Gràcia i el Raval.Treballs de Sociolingüística catalana 14 (15), 59-75. https://www.raco.cat/ index.php/TSC/article/view/38868

Sánchez Ortega, M. H. (1994). Los gitanos españoles desde su salida de la India hasta los primeros conflictos en la península. Espacio Tiempo y Forma. Serie IV, Historia Moderna ( 2), 2-7. http://revistas.uned.es/index.php/ETFIV/article/ view/3301

Schuchardt, H. (1990). Los Cantes Flamencos. Sevilla: Fundación Machado

Segre, C. (1979). La tradizione macaronica da Folengo a Gadda (e oltre). Semiotica filologica. Testo e modelli culturali, 169-83. 
Sordè, T., Flecha, R., y Mircea, T. (2013). El pueblo gitano: una identidad global sin territorio. Scripta Nova. Revista Electrónica de Geografía y Ciencias Sociales (17), 1-14. http://www.ub.edu/geocrit/sn/sn-427/sn-427-3.htm

Spivak, G. C. (2003). ¿Puede hablar el subalterno?. Revista colombiana de antropología (39), 297-364. http://www.scielo.org.co/scielo.php?script=sci_ arttext\&pid=S0486-65252003000100010

Steingress, G. (2002).Songs of the Minotaur. Hybridity and Popular Music in the Era of Globalization. A Comparative Analysis of Rebetika, Tango, Rai, Flamenco, Sardana and English Folk. Münster-Hamburg-London: LitVerlag.

Steingress, G. (2004). La hibridación transcultural como clave de la formación del Nuevo Flamenco (aspectos histórico-sociológicos, analíticos y comparativos). Trans. Revista Transcultural de Música (8), 1-25. http:/ / www.sibetrans.com/trans/ articulo/198/la-hibridacion-transcultural-como-clave-de-la-formacion-del-nuevoflamenco-aspectos-historico-sociologicos-analiticos-y-comparativos

Žižek, S. (1998). Multiculturalismo o la lógica cultural del capitalismo multinacional. En Eduardo Gruner (Ed.). Estudios culturales. Reflexiones sobre el multiculturalismo, Buenos Aires: Paidós.

--- (2008). En defensa de la intolerancia. Madrid: Sequitur. 